\title{
JPIP Proxy Server with Prefetching Strategies Based on User-Navigation Model and Semantic Map
}

\author{
J. Lino Monteagudo-Pereira, Francesc Aulí-Llinàs, Member, IEEE, and Joan Serra-Sagristà, Senior Member, IEEE
}

\begin{abstract}
The efficient transmission of large resolution images and, in particular, the interactive transmission of images in a client-server scenario, is an important aspect for many applications. Among the current image compression standards, JPEG2000 excels for its interactive transmission capabilities. In general, three mechanisms are employed to optimize the transmission of images when using the JPEG2000 Interactive Protocol (JPIP): 1) packet re-sequencing at the server; 2) prefetching at the client; and 3) proxy servers along the network infrastructure. To avoid the congestion of the network, prefetching mechanisms are not commonly employed when many clients within a local area network (LAN) browse images from a remote server. Aimed to maximize the responsiveness of all the clients within a LAN, this work proposes the use of prefetching strategies at the proxy server -rather than at the clients. The main insight behind the proposed prefetching strategies is a user-navigation model and a semantic map that predict the future requests of the clients. Experimental results indicate that the introduction of these strategies into a JPIP proxy server enhances the browsing experience of the end-users notably.
\end{abstract}

Index Terms-Interactive image transmission, JPEG2000, JPIP, prefetching strategies, user-navigation model, semantic map.

\section{INTRODUCTION}

$\mathbf{T}$ HE efficient transmission of large resolution images is a requirement in many applications related to territory management, telemedicine, disaster monitoring, or map navigation, among others. To provide a pleasant browsing experience, the transmission of the image areas requested by the users has to be optimized. Compression and scalability are fundamental aspects of the employed coding system to do so.

Among the current standards to code and transmit images, JPEG2000 excels for its coding performance and transmission capabilities. Part 1 of the standard [3] defines the core coding system, which is wavelet-based with a two tiered coding strategy. Fundamental features of JPEG2000 are support for high resolution images (in terms of spatial, spectral, or bitdepth), and scalability by spatial location, by quality, by resolution, and by component. This high degree of scalability

Copyright (c) 2013 IEEE. Personal use of this material is permitted. However, permission to use this material for any other purposes must be obtained from the IEEE by sending a request to pubs-permissions@ieee.org.

J. Lino Monteagudo-Pereira, Dr. Francesc Aulí-Llinàs, and Dr. Joan Serra-Sagristà are with the Department of Information and Communications Engineering, Universitat Autònoma de Barcelona, Spain (phone: +34 935813571; fax: +34 935814477; e-mail: jlino@deic.uab.cat, fauli@deic.uab.cat, joan.serra@uab.cat). A preliminary version of this work was presented at the IEEE Data Compression Conference 2010 and 2012 [1] [2]. This work has been partially supported by the Spanish Government (MINECO), by FEDER, and by the Catalan Government, under Grants RYC2010-05671, TIN2009-14426-C02-01, TIN2012-38102-C03-03, and 2009SGR-1224. is partially provided by a rich codestream syntax that allows random access to the file. The ability to work in the codestream domain, i.e., to identify and potentially transmit any portion of the image without needing to decode the codestream, makes JPEG2000 one of the most suitable coding standards for interactive image transmission.

The potential of JPEG2000 to interactively transmit images was explored soon after the publication of Part 1 [4]-[8]. In November 2005, JPEG2000 Part 9 [9] was published providing a versatile client-server syntax for the interchange of imagery that is named JPEG2000 Interactive Protocol (JPIP). Since then, JPIP has been adopted in fields such as medicine [10], [11], remote sensing [12], [13], or video-on-demand [14]-[16], among others.

The main asset of JPIP is the rapid recovery of the image areas requested by the client. Several mechanisms have been proposed in the literature to improve this aspect. An efficient technique employed in the server is to dismantle and resequence the original codestream [17]. Another mechanism is to utilize prefetching at the client. Prefetching refers to the ability to anticipate the future movements of the user so that the corresponding data can be retrieved during the moments in which the connection between the server and the client is idle [18]. The use of a proxy server is yet another mechanism that improves the interactive transmission capabilities of JPIP in some scenarios. Let us explain further. Commonly, in centers or institutions users browse images located in a remote server sharing the same Internet connection. In general, the channel capacity of the local area network (LAN) is much larger than that of the Internet. In such a scenario, a proxy server caches and reuses the data transmitted from the server to any of the clients, avoiding the transmission of the same information more than once. Fig. 1 illustrates this scenario. In the framework of JPEG2000, proxy mechanisms were first explored in [19] re-defining the JPIP syntax so that the conventional HTTP proxy infrastructure deployed in centers and in the Internet were able to understand the dialog between a JPIP server and a client. Unfortunately, that approach is not compliant with the standard. The first compliant JPIP proxy server was introduced in [20] employing re-sequencing techniques similar to those of [17] to optimize the delivery of data to the clients in the LAN.

As seen in the experiments reported in Section V, it is not recommendable that the clients use prefetching individually in the scenario of Fig. 1 because that might saturate the Internet connection. None of the JPIP proxy servers proposed in the literature employs prefetching strategies, either. So, typically, the Internet connection $\{B, C\}$ depicted in Fig. 1 


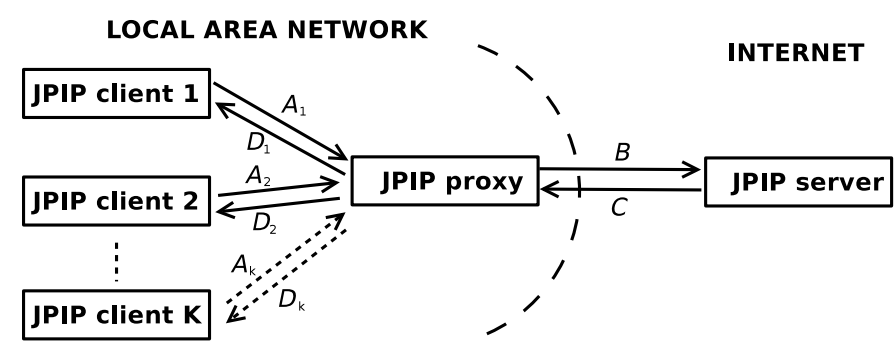

Fig. 1: JPIP clients within a LAN share the same Internet connection to browse images located in a remote JPIP server. Data transmitted from the server to the clients is cached and reused by a JPIP proxy server.

is left idle when users have retrieved the requested areas. The purpose of this work is to introduce prefetching strategies in the JPIP proxy server -rather than to each client- to enhance the browsing experience of the clients within the LAN. The JPIP proxy server proposed herein employs a rate-distortion optimized algorithm that maximizes the responsiveness of the whole system taking into account the current and (possible) future areas requested by the clients. The functionality of the proposed algorithm is the same if the proxy is introduced in scenarios with a more complex topology than that of Fig. 1. Prefetching is carried out only by the proxy when the Internet connection is idle. Two strategies are put in practice to predict the areas that the clients may request in the future. The first employs a user-navigation model that has been extracted from the logs collected by several image servers belonging to the remote sensing community. The second strategy utilizes a semantic map that prioritizes areas of the image depending on their content. Experimental results report significant gains with respect to classical client-server approaches and with respect to conventional JPIP proxy servers without prefetching strategies. The research of this paper extends our previous works [1], [2] by describing a more accurate user-navigation model, the novel semantic map-based prefetching strategy, and by providing extended experimental results.

The paper is structured as follows. Section II reviews fundamentals concepts of the JPEG2000 core coding system, the codestream organization, and the JPIP protocol. The architecture and functionalities of the proposed JPIP proxy server are described in Section III, whereas Section IV introduces the two prefetching strategies proposed. The performance achieved by our JPIP proxy server and other conventional transmission strategies is assessed in Section V through experimental results carried out for large remote sensing images. The last section concludes this work with a brief summary and some remarks.

\section{OVERVIEW OF JPEG2000 AND JPIP}

The JPEG2000 core coding system is constituted by four main coding stages [21]: sample data transformation, sample data coding (tier-1), rate-distortion optimization, and codestream re-organization (tier-2). The first stage applies a wavelet transform that decorrelates and decomposes the image in successive levels of resolution. Then, the image is conceptually partitioned in small sets of wavelet coefficients, called codeblocks, that are coded by the tier- 1 coding stage by means of a bitplane coding engine. Tier- 1 produces a quality embedded bitstream for each codeblock that can be truncated at increasing rates. The third stage of the coding system forms quality layers using rate-distortion optimization techniques. A quality layer is defined as a collection of bitstream segments from different codeblocks. Its transmission and/or decoding represents an increment on the quality of the image. Commonly, the ratedistortion optimization stage employs Lagrange optimization to minimize the distortion at the target rates selected for the quality layers and for the final codestream. To do so, first the convex hull of individual codeblocks is established by identifying those truncation points with strictly decreasing distortion-rate slope. If $d_{n}$ and $r_{n}$ respectively denote the distortion and the rate achieved at the truncation points of the bitstream generated for a codeblock, the distortion-rate slope is defined as $s_{n}=\frac{d_{n-1}-d_{n}}{r_{n}-r_{n-1}}$. Quality layers are then formed selecting bitstream segments from the union of all codeblocks with the highest distortion-rate slopes. Through this process, each layer contains bitstream segments with equal or higher distortion-rate slope than the threshold achieved for that layer, which is referred to as $S_{q}$ with $1 \leq q \leq Q$, $Q$ denoting the number of layers of the codestream. The last stage of the coding pipeline is the tier-2, which codes auxiliary information. In this and following discussions we assume that the image is not partitioned in tiles [21, Ch. 11.2] since interactive transmission is commonly not benefited from such partitions [5].

As depicted in Fig. 2, the JPEG2000 codestream is structured in containers. The smallest container is the packet, which encapsulates segments of the bitstreams generated from codeblocks that belong to the same component, resolution level, and spatial area of the image, the so-called precinct. Data produced for each precinct are distributed in as many packets (some of them possibly null) as quality layers has the final codestream. When the image is interactively transmitted, JPIP servers generally dismantle the codestream into a collection of data-bins that contain all packets belonging to one precinct. When the client requests an image region, called window of interest (WOI), the server identifies those data-bins containing encoded data from that WOI and transmits segments of these data-bins to the client. Rather than to interact directly with the codestream, JPIP defines a versatile dialog between client and server in which the client requests a WOI specifying spatial location, resolution, and components on the image domain, and the server identifies that WOI in the compressed domain. Messages containing one or several segments of data-bins are referred to as JPP-streams.

As mentioned above, an important issue that JPIP servers contemplate is the delivery of data in an optimized fashion. Let us denote the packets of the codestream as $\mathcal{T}_{c, r, p, q}$, where subindexes $c, r, p, q$ identify the component, resolution level, spatial location, and quality layer of the packet, respectively. When the client requests a WOI, say $\mathcal{W}$, the server identifies the packets related to the WOI, which we denote as $\mathcal{T}_{c, r, p, q} \succ$ $\mathcal{W}$, and determines adequate rates for JPP-streams transmitted to the client.

To optimize the rate-distortion efficiency, the server com- 


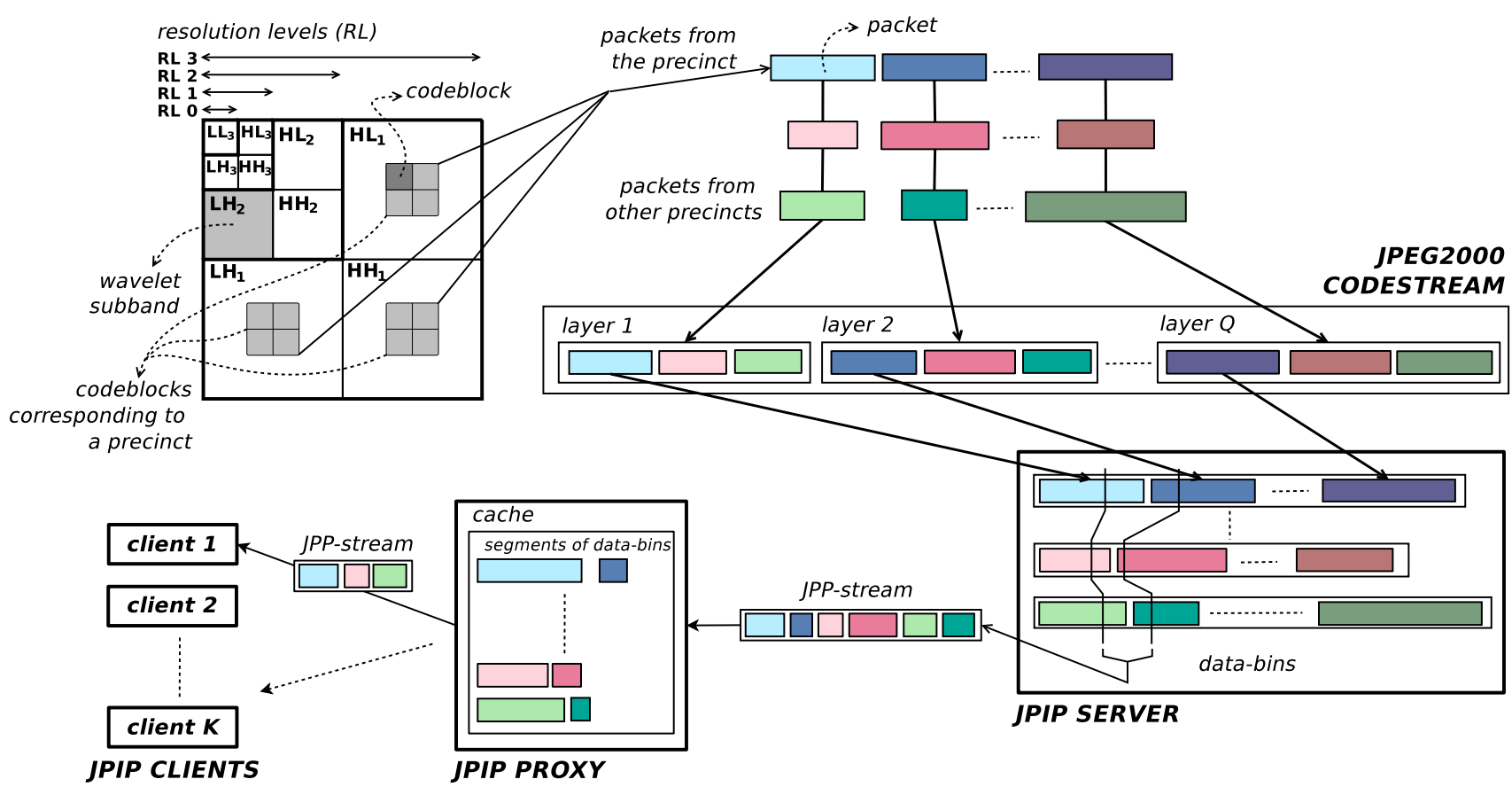

Fig. 2: Simplified overview of the JPEG2000 partitioning system, the organization of the codestream, and the data transmitted by the JPIP server, proxy, and client.

monly selects packets according to the layer's order, i.e., packets in first quality layers are transmitted first. Since $\mathcal{W}$ does not typically match exactly with image precincts, [17] suggested the application of a window scaling factor $F_{c, r, p} \in(0,1]$ that accounts for the percentage of coefficients within that precinct that are relevant to $\mathcal{W}$. Through $F_{c, r, p}$, data is re-sequenced and transmitted to the client according to artificial distortionrate slopes computed for individual packets as

$$
S_{c, r, p, q}^{\prime}=S_{q} \cdot F_{c, r, p} .
$$

This re-sequenced packet ordering does not assure the minimization of distortion per transmitted unit since $S_{q}$ is only an approximation of the real distortion-rate slope for packet $\mathcal{T}_{c, r, p, q}$. The real distortion-rate slope of $\mathcal{T}_{c, r, p, q}$ is not stored in the codestream because it requires a significant rate and is not necessary to decode the image. This poses an issue that has been approached in the literature from different points of view [14], [17], [18], [22], [23]. As reported in these works, the use of $S_{q}$ in Equation 1 provides near optimal performance in practice. As common, we assume that values for $S_{q}$ are recorded in the codestream.

All data transmitted by the server is cached by the client. The server can maintain a model of the client's cache to avoid the retransmission of the same data. In this case the connection is named stateful. When the server does not maintain the model of the client's cache, the client may include a description of its cache when requesting WOIs. In this case, the connection is named stateless. Of the two, stateful connections are more convenient to minimize the transmission of side information [24].

\section{Proposed JPIP Proxy Server}

\section{A. System architecture}

The proposed JPIP proxy server maintains the full set of JPIP features, keeping compliance with the standard. The main ability of the proxy is to understand the JPIP protocol so that it can dismantle and cache the JPP-streams transmitted by the server as a collection of data-bins (see Fig. 2). These data-bins are associated to packets in the codestream and to precincts in the image domain, which allows their optimized re-sequencing to respond the clients' requests. To replace the server efficiently, the JPIP proxy also maintains a model of the clients' cache to allow stateful connections.

As observed by many authors [18], [25], [26], there exists a delay between the emission of data by the server and the feedback provided by the user before requesting a new WOI. This delay includes the transmission time, decoding latency, and the time that the user needs to interpret newly received visual information. During this time, the connection between the client and the server is idle. As stated before, idle moments can be employed to prefetch data that may be requested in the future by the client(s). In the scenario of Fig. 1, prefetching should not be directly applied to all clients within a LAN as formulated in [18] because the joint transmission of WOIs that are in current use, with WOIs that might possibly be required, could cause the assignment of the available channel resources to useless queries, providing negligible gains on the overall system performance, as is experimentally demonstrated in Section V.

Our approach introduces prefetching strategies in the JPIP proxy server combining techniques of rate-distortion optimization with a model of probabilities that predicts the next users' movements. The proxy provides prefetching to all clients 
within the LAN simultaneously. The proposed proxy server has two modes of operation: normal and prefetching. The normal mode is active when at least one client requests a WOI that is not fully contained in the proxy's cache. The prefetching mode is active when all client's petitions can be responded with the cached data, and so the connection between the proxy and the server is idle.

The functions that are carried out by the proxy server when it is in normal mode are similar to those of a conventional proxy [20]. When a client within the LAN requests a WOI to the JPIP proxy server (link $A_{k}$ in Fig. 1), the proxy checks whether that WOI is contained in its cache or not. In the case that there are no data-bins belonging to the requested WOI in the cache, the proxy sends the petition to the server (connection $\{B, C\}$ ), and responds to the client caching all data transmitted from the server. In the case that the WOI is partially contained in the cache, the proxy transmits cached data to the client and, simultaneously, re-formulates the petition to the server requesting non-cached data. In the case that the WOI is totally contained in the cache, the proxy responds to the client transmitting JPP-streams (link $D_{k}$ ). Evidently, the proxy can handle more than one request at the same time, so this procedure is executed in parallel for all concurrent requests.

To minimize the interchange of image data between the proxy and the server when the proxy is in normal mode, WOIs requested to the server are re-formulated. Rather than including several areas demanded by clients as in [20], our proxy requests specific WOIs one by one in the order that maximizes the overall system's performance. Let us extend the notation of the previous section to denote the WOIs currently requested by the clients as $\mathcal{W}_{k}$, with $1 \leq k \leq K, K$ being the number of active clients. In normal mode, the proxy computes artificial distortion-rate slopes $S_{c, r, p, q}^{\prime \prime}$ for packets considering all clients browsing the same image according to

$$
S_{c, r, p, q}^{\prime \prime}=S_{q} \cdot F_{c, r, p}^{\prime} \cdot \frac{1}{K} \sum_{k} \delta\left(\mathcal{T}_{c, r, p, q}, k\right) \forall c, r, p, q,
$$

with

$$
\delta\left(\mathcal{T}_{c, r, p, q}, k\right)= \begin{cases}1 & \text { if } \mathcal{T}_{c, r, p, q} \succ \mathcal{W}_{k} . \\ 0 & \text { otherwise }\end{cases}
$$

$\delta\left(\mathcal{T}_{c, r, p, q}, k\right)$ is a binary function that ascertains whether packet $\mathcal{T}_{c, r, p, q}$ is necessary to serve the current WOI $\mathcal{W}_{k}$ requested by client $k$. When a packet is necessary to serve the WOIs from all the clients, $\frac{1}{K} \sum_{k} \delta\left(\mathcal{T}_{c, r, p, q}, k\right)$ in (2) results in 1. Otherwise, it results in the fraction of clients requesting that packet. $F_{c, r, p}^{\prime} \in(0,1]$ in Equation (2) accounts for the percentage of coefficients in the precinct that are relevant to the WOIs of all clients. We note that $S_{c, r, p, q}^{\prime \prime}$ is computed for all the packets of the codestream.

Packets are requested by the proxy in decreasing order of $S_{c, r, p, q}^{\prime \prime}$. This strategy maximizes the overall system's performance since the highest $S_{c, r, p, q}^{\prime \prime}$ corresponds to that packet

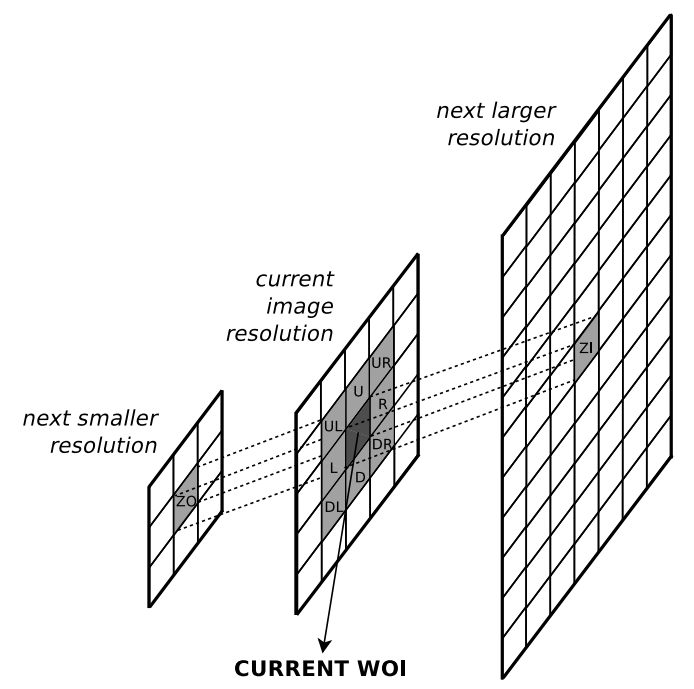

Fig. 3: Partitioning system of a multi-resolution image originating from the current WOI. The possible movements of a user in future requests are labeled as: $\mathrm{U}$ (up), UR (up right), $\mathrm{R}$ (right), DR (down right), D (down), DL (down left), L (left), UL (up left), ZI (zoom in), and ZO (zoom out).

that minimizes the clients' distortion per transmitted unit in link $C$ of Fig. 1. Once the packet arrives to the proxy, it is transmitted to the clients using artificial slopes $S_{c, r, p, q}^{\prime}$ to form JPP-streams, thus maximizing the performance in $D_{k}$ links.

In prefetching mode, the JPIP proxy server requests WOIs that may be demanded in the future by the clients. Although most browsing applications allow the user to request any spatial area of the image at any of the available resolutions, the evaluation carried out in Section IV-A indicates that aimless movements are only 5\% of all movements executed in a normal browsing session, being more usual those movements that displace the active view to areas adjacent to the current WOI. Furthermore, the resolution of the active view -not the resolution of the image browsed-is seldom changed by the user, being approximately only $3 \%$ of all movements executed in a browsing session as indicated below. These observations are employed to define the coordinate system of the potential future WOIs requested by the clients.

The idea is that once the user has requested the first WOI, the whole image -including all available resolutions- can be partitioned in equally sized regions with origin on, and dimensions of, that first WOI. This partitioning system, which is depicted in Fig. 3, eases the identification of the areas adjacent to the current WOI that are susceptible to be requested in next commands. Evidently, the partitioning is carried out particularly for each user. If the size of the requested WOI changes, the image needs to be re-partitioned. But this does not happen often in practice and, even if it does occur, it does not affect the proposed model since only individual movements from one WOI to the next are considered. These areas are depicted in gray in the figure. There are ten next possible movements corresponding to eight panning operations (vertical and horizontal displacements in the same resolution level), and two zooming operations (changes on the image 
resolution). We note that the "zoom in" operation may consider not only the central region of the current WOI in the next larger resolution (as it is depicted in Fig. 3) but all regions at the larger resolution level that correspond to the current WOI. Our partitioning system considers only the central region due to the empirical observations reported in Section IV-A indicate that that is the most frequent "zoom in" operation carried out by users.

The movements carried out by the user are denoted as $X$, with $X=\{$ up $\mid$ up right $\mid$ right $\mid$ down right $\mid$ down | down left $\mid$ left $\mid$ up left $\mid$ zoom in $\mid$ zoom out $\}$. The WOIs that the clients can request in the future are defined as a function of the current WOI $\mathcal{W}_{k}$ and a user movement $X$ as $f\left(\mathcal{W}_{k}, X\right)$. Evidently, $f(\cdot)$ always returns a WOI side by side $\mathcal{W}_{k}$ (including different resolutions) since $X$ is defined only as adjacent movements. When the JPIP proxy server is in prefetching mode, artificial distortion-rate slopes $S_{c, r, p, q}^{\prime \prime \prime}$ are computed as

$$
S_{c, r, p, q}^{\prime \prime \prime}=S_{q} \cdot F_{c, r, p}^{\prime} \cdot \frac{1}{K} \sum_{k} \delta^{\prime}\left(\mathcal{T}_{c, r, p, q}, k\right) \forall c, r, p, q,
$$

with

$$
\delta^{\prime}\left(\mathcal{T}_{c, r, p, q}, k\right)=\sum_{X}\left\{\begin{array}{ll}
P(X) & \text { if } \mathcal{T}_{c, r, p, q} \succ f\left(\mathcal{W}_{k}, X\right) \\
0 & \text { otherwise }
\end{array} .\right.
$$

Equation (4) is equal to (2) except for the use of $\delta^{\prime}(\cdot) . \delta^{\prime}(\cdot)$ is a function that returns the probability of packet $\mathcal{T}_{c, r, p, q}$ to be requested by client $k$ in future movements. The summation in Equation (5) accounts for the probabilities of all possible future movements of the client with regard to that packet. $P(X)$ in (5) is the probability that the user executes the movement in future requests. It is determined through the strategies described in the next section. When the proxy is in prefetching mode, it requests WOIs in decreasing order of $S_{c, r, p, q}^{\prime \prime \prime}$ and caches the response hoping that future user movements will request prefetched data. Prefetched data are kept in cache even when the prediction fails because other users may browse that area of the image in future requests.

Briefly summarized, the functions carried out by the JPIP proxy server are the delivery of data to the clients through links $D_{k}$ using $S_{c, r, p, q}^{\prime}$, and the requesting of WOIs to the server through link $B$ using $S_{c, r, p, q}^{\prime \prime}$ when the proxy is in normal mode, and using $S_{c, r, p, q}^{\prime \prime \prime}$ when the proxy is in prefetching mode. As in [17], [20], strict optimal performance is not guaranteed due to the use of artificial distortion-rate slope for packets.

\section{B. Implementation considerations}

Some considerations have to be taken into account to implement the proposed JPIP proxy server:

- The connection $\{B, C\}$ between proxy and server must use the JPIP options ALIGN and AUX. ALIGN forces the JPIP server to deliver non-segmented packets, which is required by the proxy to identify individual packets
$\mathcal{T}_{c, r, p, q}$ without needing to decode data. AUX forces the JPIP server to include a field that specifies the layers to which packets belong to. These options are not required in links $A_{k}, D_{k}$.

- The JPIP proxy server requests WOIs (link $B$ ) specifying the number of layers through the JPIP option LAYERS. This is mandatory to force the server to transmit a specific number of layers for the requested precinct. Note that this blocks any rate control mechanism used in the server to transmit data, intentionally leaving the control to the proxy. Although this causes requests of multiple WOIs in a short period of time, the results reported in Section V indicate that this strategy does not saturate the network link to the server, working efficiently in practice. To force the server to complete the transmission of requested WOIs, the proxy must include the parameter WAIT in requests. This assures that the delivery of packets is carried out as intended by the proxy and that transmissions are not interrupted as more requests arrive to the server.

- We assume that values for $S_{q}$ are available to the proxy through their transmission in COM markers of the codestream, or through rough estimations as described in [27].

- As seen in Fig. 1, the server sees the proxy as a single client, thus the use of a stateful connection is highly recommended in connection $\{B, C\}$. Connections between the proxy and clients can be stateless.

- The operations carried out by the proxy do not entail the re-encoding of any part of the codestream, so the system can be escalated with the proxy handling many clients simultaneously [20].

- The administration of the LAN must consider that a failure in the proxy server is critic since it leaves all clients without connection. This may be solved using a high availability cluster, for example.

\section{Prefetching Strategies}

The goal of the JPIP proxy server when it is in prefetching mode is to optimize the responsiveness of all clients. Key to achieve this goal is to foresee the WOIs that are more likely to be requested by the clients. The objective is then to obtain a precise model for probabilities $P(X)$ employed in Equation (5). Two approaches are used. The first is based on a user-navigation model that captures the common behavior of users when navigating on an image. The second approach utilizes the content of the image to predict the probability of an image area to be visited more or less frequently. The former approach can be employed when the content of the image is not known or when it is too arduous to determine, whereas the latter can be employed otherwise.

\section{A. User-navigation model}

The user-navigation model is described first. The main insight behind this approach is to observe the chain of movements carried out by users when navigating on an image. The individual probabilities of the 10 movements defined in Fig. 3 are obtained as follows. The first step was to record WOIs requested by clients when browsing four large resolution 


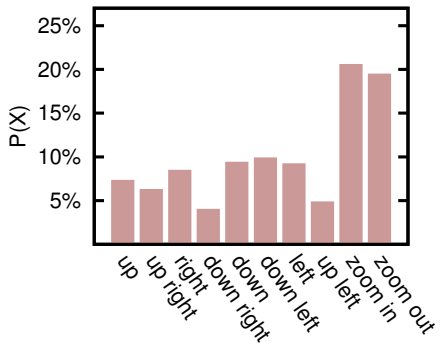

(a)

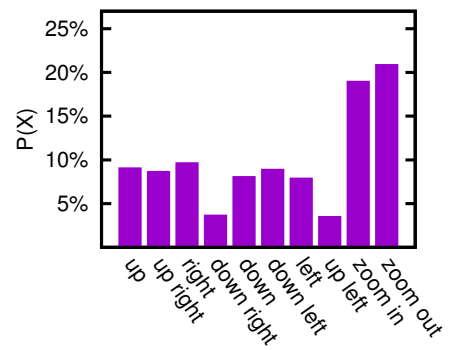

(b)

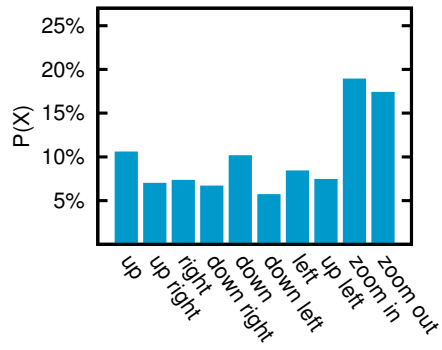

(c)

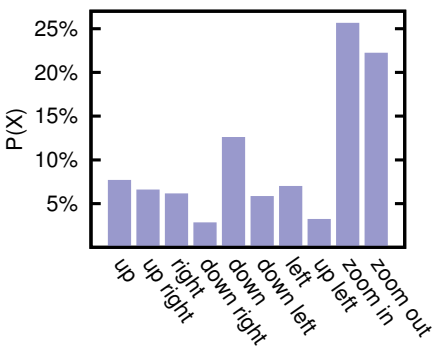

(d)

Fig. 4: Evaluation of the movements carried out by users when navigating on the images of the remote sensing corpus (a) "Barcelona" 1629 sessions, (b) "Girona" 1203 sessions, (c) "Lleida" 923 sessions, and (d) "Catalunya" 228016 sessions.

images during a period of approximately 3 years. Images employed in this experiment are referred to as "Barcelona", "Girona", "Lleida", and "Catalunya", and are available with a large variety of resolution sizes in the server. The size of the largest resolution is approximately $13000 \times 13000$ pixels. The images belong to the remote sensing community and cover large areas of Catalunya (a region of Spain). They are browsed through MiraMon, which is a popular remote sensing application developed by CREAF [28]. In general, the size of the user's view is set to $800 \times 600$ pixels in this application.

To analyze only browsing commands relevant to our purposes, the second step of this analysis is to identify the socalled sessions in the logs gathered by the server. One session collects all commands executed by one client considering a maximum time interval of 30 minutes between consecutive requests. If two consecutive commands are delayed in more than 30 minutes, they are considered as two different sessions. This time window gives spare time to the user to receive and observe the requested WOI. Only sessions with more than three movements are considered; the remaining are irrelevant to our purposes and are discarded. In total, the selected sessions accumulate 1629, 1203, 923, and 228016 client requests for "Barcelona", "Girona", "Lleida", and "Catalunya", respectively.

The third step of the analysis is to translate the relevant WOI requests into the ten movements defined by $X$. Movements that do not correspond to any of these categories represent less than $8 \%$ of all requests analyzed and are discarded. The discarded movements correspond to changes on the resolution of the active view, or aimless movements. On average, the number of movements in each session is approximately 13 . The average time that the users spend on a browsing session is 7 minutes, approximately. The probability of user movements (i.e., $P(X)$ ) is determined for each image considering all commands of all sessions. Fig. 4 reports the individual probabilities achieved for $P(X)$, for the four images. Results suggest that users navigate similarly on images "Barcelona", "Girona", and "Lleida". For these images, the navigational commands that are most frequent correspond to the zooming operations, which have a probability of $40 \%$ to be executed, approximately. There is no significant difference between "zoom in" and "zoom out". The remaining movements corresponding to panning operations present slight differences among the three images, though they are not significant. Our experience indicates that $P^{\prime}(X)=$ $20 \%$ when $X=\{$ zoom in $\mid$ zoom out $\}$ and $P^{\prime}(X)=$ $7.5 \%$ when $X=\{$ up $\mid$ up right $\mid$ right $\mid$ down right $\mid$ down $\mid$ down left $\mid$ left $\mid$ up left $\}$ is a model that, in this context, captures well the behavior of most users browsing an image. Finer adjustment of these probabilities does not seem to improve performance significantly. The use of conditional probabilities considering the last movement of the user to predict the next one (not shown in the figures) does not provide any significant gain either.

The experimental results reported in Fig. 4(d) for "Catalunya" suggest that the users have a slightly different behavior when navigating on this image. The main difference compared with the previous images is that the users move more frequently downward than upward. More precisely, the probability of panning movements $X=$ \{ down right $\mid$ down $\mid$ down left $\}$ is $22 \%$ against the $17 \%$ achieved for $X=\{$ up left $\mid$ up $\mid$ up right $\}$. Such a difference is only found in this image. It is caused due to the positioning of the first WOI, which is fixed by the server at the top of the image. This compels most users to reach their areas of interest moving downward. Also, the zooming operations carried out on this image have a slightly higher probability of being executed than that found in the previous three images. This may be caused due to the high spatial resolution available for this image, which reaches 10 meters per pixel at the highest resolution level. This may trigger the curiosity of the users to zoom in and out more frequently to explore the requested areas in more detail. As seen in Section V, a slight increment on performance is achieved for this image when probabilities $P(X)$ correspond to those reported in Fig. 4(d) (i.e., $P^{\prime \prime}(X)=\{7.7 \%|6.6 \%| 6.2 \%|2.9 \%| 12.6 \%|5.9 \%| 7 \% \mid$ $3.3 \%|25.7 \%| 22.3 \%\}$ in the movement order reported in the figure). We remark that these observations result in a slight change in the model that has little impact on the performance of the system. The user-navigation models employing $P^{\prime}(X)$ and $P^{\prime \prime}(X)$ mainly describe the same underlying user behavior.

The user-navigation model determined in this analysis is valid for applications that transmit interactively remote sensing 


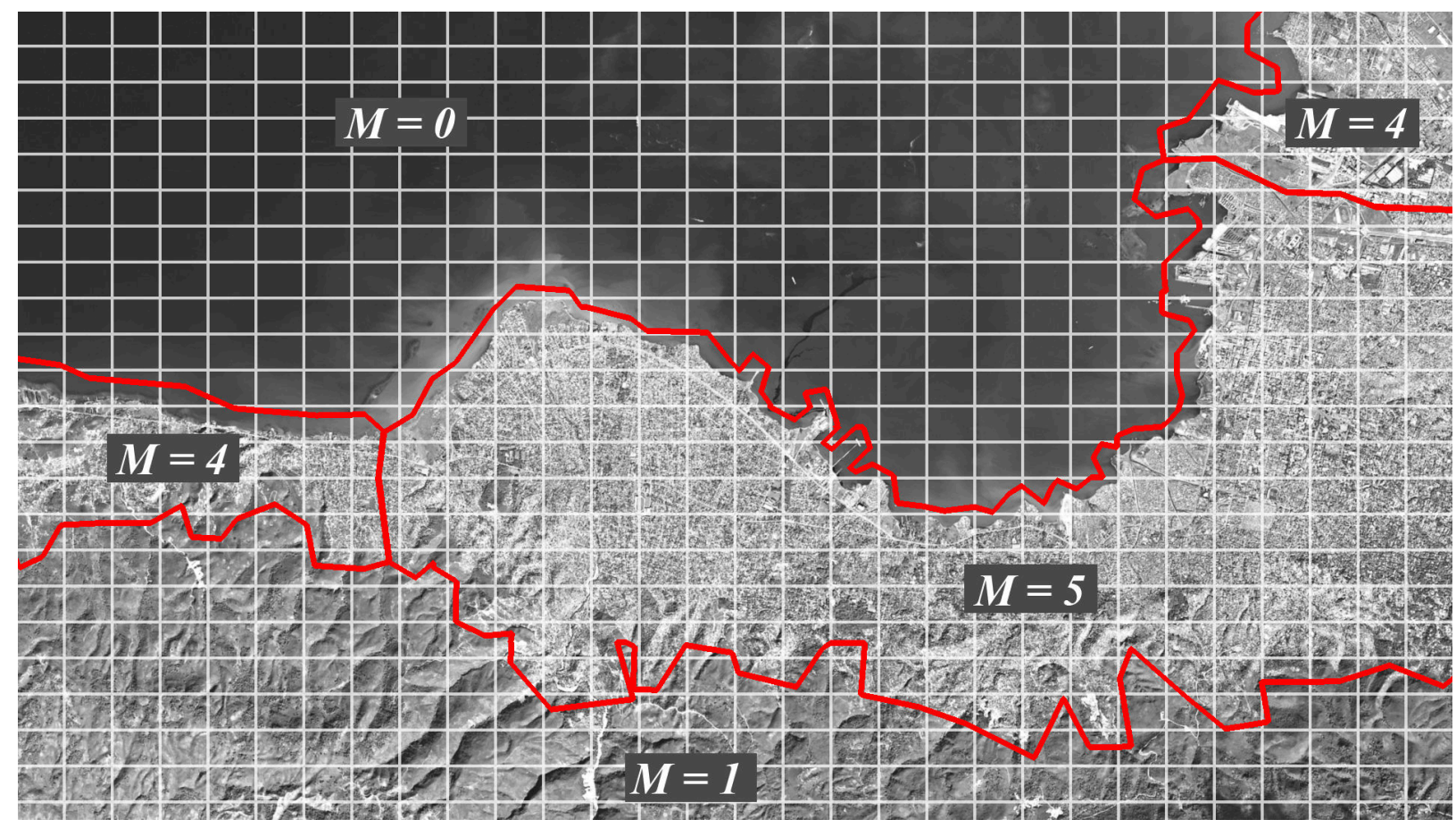

Fig. 5: Semantic map and precincts employed for the "Port-au-Prince city" image (see the details of this image in Section V). The semantic regions are delimited in red. The label on each region indicates its priority, which is denoted as $M$ and is determined depending on the content of the image. The areas corresponding to the city center and its surroundings have the highest priorities, whereas the forest and the sea have lower priorities. The white lines forming the grid correspond to the JPEG2000 precincts of resolution level 3 (resolution level 0 and 5 are the smallest and the largest, respectively).

images of high resolution. Other applications that transmit images with different particularities and/or users behaving differently may require different models. The methodology described above can be employed in other scenarios to determine suitable user-navigation models for them.

\section{B. Semantic map}

The content of the image is also a good indicator for the probability of an area to be visited. This observation has been used recently by the remote sensing community with the aim to optimize the computational resources of a web map server [26]. To the best of our knowledge, such an approach has never been employed in the framework of JPIP. The main idea is to assign high probabilities to those movements that displace the WOI to image areas whose contents are more appealing to the user. This strategy is implemented in the JPIP proxy server as follows. First, the image is partitioned in regions containing different types of content. In a remote sensing image, these regions may correspond to cities, roads, seas, or deserted areas, for instance. As stated in [26], each type of region has a different chance to be visited. Cities are more commonly browsed by users than deserted areas, for example. So selected priorities are assigned to each region type. The higher the priority, the higher the chance that that image area is visited. Depending on how this operation is implemented, it may require the supervision of an expert in the field, or the use of other techniques such as classification [29]. It is important to employ accurate methods of supervision/classification since otherwise the performance of the system may be penalized.
The selected regions together with their priorities form the so-called semantic map. The semantic map is employed to obtain individual priorities for packets. As described above, packets $\mathcal{T}_{c, r, p, q}$ are quality increments corresponding to the precinct of component $c$, resolution level $r$, and spatial location $p$. Fig. 5 illustrates the relation between the semantic map and the precincts of one resolution level of an image employed in the experimental tests of the next section. As seen in the figure, each precinct may be contained in one or more semantic regions. When a precinct is fully contained in one region, the priority of that region is assigned to all packets of that precinct. When a precinct is partially contained in more than one region, the priority for packets of that precinct is computed as a weighted average among the regions to which it belongs. More precisely, probabilities $P(X)$ employed in Equation (5) are determined according to

$$
P^{\prime \prime \prime}(X)=\frac{\delta^{\prime \prime}\left(\mathcal{T}_{c, r, p, q}, X\right)}{\sum_{X^{\prime}} \delta^{\prime \prime}\left(\mathcal{T}_{c, r, p, q}, X^{\prime}\right)}
$$

with

$$
\begin{gathered}
\delta^{\prime \prime}\left(\mathcal{T}_{c, r, p, q}, X\right)= \\
\begin{cases}\sum_{s} F_{c, r, p, e}^{\prime \prime} \cdot M_{c, r, p, e} & \text { if } \mathcal{T}_{c, r, p, q} \succ f\left(\mathcal{W}_{k}, X\right) \\
0 & \text { otherwise }\end{cases}
\end{gathered}
$$


$\delta^{\prime \prime}(\cdot)$ is a function that returns the priority assigned through the semantic map to packet $\mathcal{T}_{c, r, p, q}$ when the user executes movement $X$. When the packet is related to WOI $\mathcal{W}_{k}$, this priority is computed in Equation (7) as $\sum_{s} F_{c, r, p, e}^{\prime \prime} \cdot M_{c, r, p, e}$. $M_{c, r, p, e}$, with $1 \leq e \leq E$, denotes the priorities of the $E$ semantic regions that correspond to the precinct located at $c, r, p . F_{c, r, p, e}^{\prime \prime}$ accounts for the percentage of coefficients within the precinct located at $c, r, p$ that are relevant to the semantic region $e$. Equation (6) computes $P(X)$ as the priority of movement $X$ divided by the summation of priorities of all possible movements.

\section{EXPERIMENTAL RESULTS}

All the infrastructure used to evaluate the proposed method, including the client, the server, and the JPIP proxy server are implemented in our JPEG2000 Part 9 implementation CADI [30]. Experiments are carried out in a LAN with a channel capacity of $100 \mathrm{Mbps}$ and a connection to the Internet shared by all clients with a channel capacity of $10 \mathrm{Mbps}$. The images employed in the experiments are two satellite images from GeoEye Inc. that were provided to help missions responding to the earthquake that struck Haiti in 2010. Our aim is that rescue teams in similar situations employ the proposed mechanisms to enhance their transmission infrastructure. The images cover areas of Port-au-Prince and its surroundings, are referred to as "Port-au-Prince airport" and "Port-au-Prince city", and are 8 -bit gray scale with size $32768 \times 19456$ and $31744 \times 20480$, respectively. The 8-bit gray scale Landsat image "Catalunya" utilized in Section IV-A is also employed in the experiments. It is provided by CREAF and has a size of $13561 \times 13161$. These images are compressed with JPEG2000 using the following coding parameters: 5 levels of irreversible CDF 9/7 wavelet transform, codeblock size of $64 \times 64$, restart coding variation, and 25 quality layers logarithmically spaced in terms of bitrate. The server adjusts on-the-fly the precinct size in all resolution levels according to the codeblock size to enhance interactivity options.

Results are obtained when four or five users browse an image simultaneously. Eight transmission strategies are evaluated along the following experiments:

1) Client-server: the image transmission is carried out without using any proxy infrastructure.

2) Client-server with prefetching at the clients: the image transmission is carried out without using any proxy infrastructure, and prefetching is employed individually at each client.

3) Conventional JPIP proxy: all data transmitted between the server and the clients are cached by a proxy that reuses already transmitted packets. This strategy is (almost) equivalent to that introduced in [20].

4) Conventional JPIP proxy with prefetching at the clients: prefetching is employed individually at each client and all data transmitted between the server and the clients are cached by a proxy.

5) JPIP proxy with simple prefetching: this strategy uses the proxy described in Section III but employing a uniform model of probabilities for the prefetching mode, i.e.,
$P(X)=1 / 10 \forall X$. This strategy was introduced in our previous work [1]. Here, it serves to appraise the gain that is achieved when prefetching is carried out employing the probability models described in Section IV.

6) JPIP proxy with prefetching based on the usernavigation model: this strategy uses the proxy described in Section III with prefetching based on the usernavigation model described in Section IV-A. Employed probabilities are $P^{\prime}(X)$ or $P^{\prime \prime}(X)$ as indicated.

7) JPIP proxy with prefetching based on the usernavigation model and Quality of Service $(Q o S)$ considerations: this strategy is as the previous one except for the consideration of clients that may have a higher priority during the prefetching mode of the proxy (see below).

8) JPIP proxy with prefetching based on the semantic map: this strategy uses the proxy described in Section III with prefetching based on the semantic map described in Section IV-B. Employed probabilities are $P^{\prime \prime \prime}(X)$.

The results are reported for these strategies when the clients execute their browsing sessions identically. Evidently, each client browse different areas of the image, but the same chain of movements is reproduced by each client when evaluating different strategies. The chain of movements selected for each user corresponds to typical browsing sessions exploring areas of an image. We allow users to perform up to 13 movements during a session that lasts 7 minutes approximately. This is a behavior typically observed in browsing sessions, as described in Section IV-A.

Fig. 6 reports the results achieved when four clients browse the "Port-au-Prince airport" image. The vertical axis of these and following figures is the quality of the retrieved WOI, in terms of Peak Signal to Noise Ratio (PSNR). The horizontal axis is the browsing session time. Only three representative WOIs selected for each client are reported in these figures for the sake of clarity. The WOIs correspond to three consecutive movements of the user and are selected to illustrate the behavior of each strategy. Similar results hold for the remaining -not reported- WOIs. To enhance the presentation, the horizontal axes of the figures are broken between WOIs. All plots starting from the same point, approximately, correspond to the same WOI retrieved by the client. WOIs are decoded at the end of each quality layer, hence, the quality achieved by all strategies when decoding the same WOI is equal, changing only the instant of time at which the layer is completely available at the client. Fig. 6 reports results for five of the strategies described before, namely, client-server, conventional JPIP proxy, JPIP proxy with simple prefetching, JPIP proxy with prefetching based on the user-navigation model, and JPIP proxy with prefetching based on the user-navigation model and QoS considerations.

In general, the WOIs requested by the clients at the beginning of their sessions are recovered similarly by all strategies since there are no data in the cache of the proxy. See, for instance, in Fig. 6(c) that all transmission strategies recover WOI 2 of client 3 at the same pace. As the browsing session advances, transmission strategies that employ proxy infrastructure recover WOIs more rapidly than the client-server strategy due to data already transmitted for other clients can 


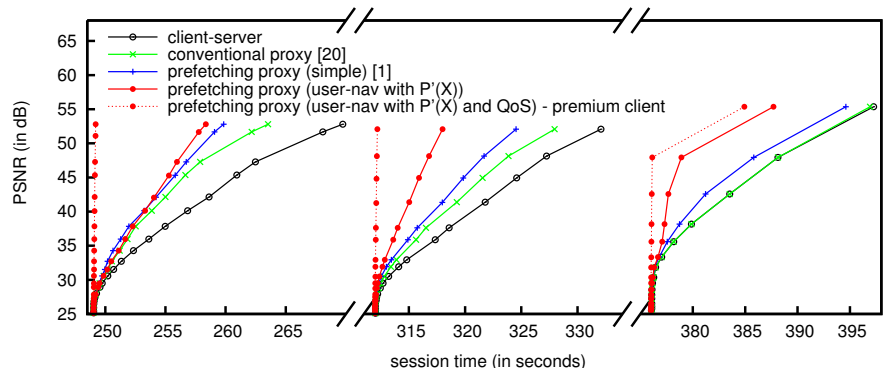

(a)

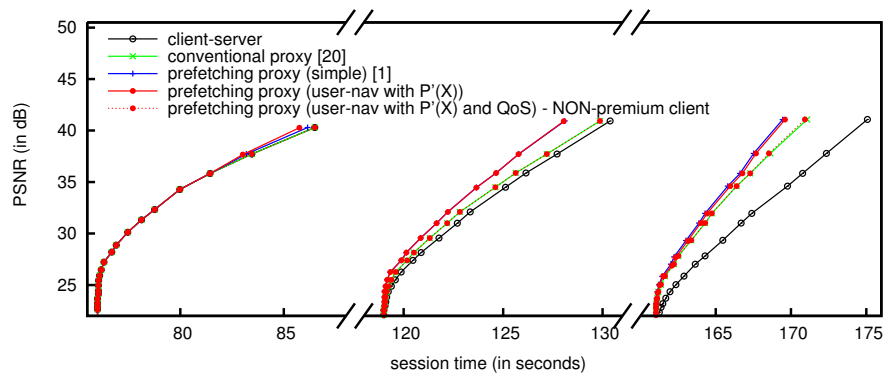

(c)

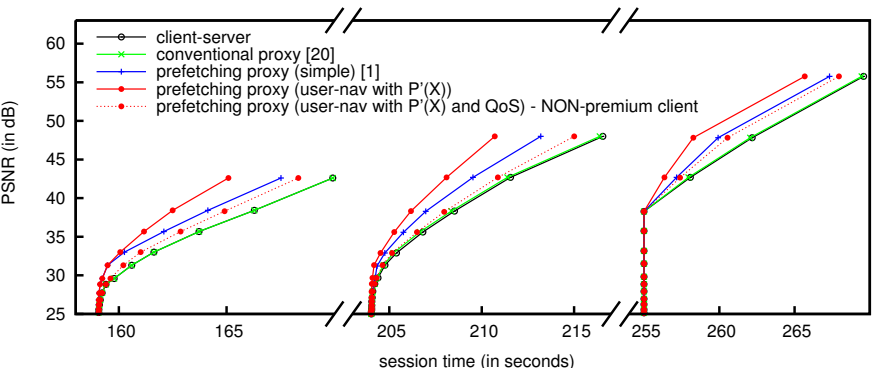

(b)

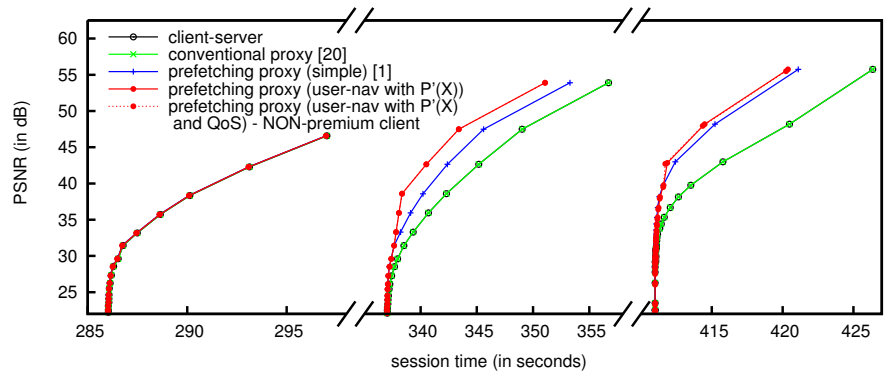

(d)

Fig. 6: Evaluation of the performance achieved by a proxy server with a prefetching strategy based on a user-navigation model compared to three other strategies. The image browsed is "Port-au-Prince airport". The following WOIs are reported: (a) 5, 6, and 7 from client 1; (b) 5, 6, and 7 from client 2; (c) 2, 3, and 4 from client 3; and (d) 6, 7, and 8 from client 4.

be reused. This is seen in WOIs 3 and 4 requested by client 3 (Fig. 6(c)). Although it is not usual, in some occasions a WOI requested in the middle, or at the end, of a session is also recovered by all strategies similarly. See, for instance, WOI 6 requested by client 4 in Fig. 6(d). This happens only when the user moves to an area that was neither browsed by other clients nor prefetched by the proxy. As seen in Fig. 6, the most typical behavior is that the proxy using prefetching strategies based on the user-navigation model serves the WOI more rapidly than the other strategies. Note, for example, that the last WOI reported in Fig. 6(a) is delivered by our proxy to the client almost 10 seconds before all other transmission strategies. The differences between the conventional proxy and the proxies using prefetching become evident after the second minute of the session (not shown in the figure), approximately, since there have been enough idle moments to prefetch more data from the server. As seen in the figure, the differences are relevant for some WOIs (e.g., second and third WOI reported in Fig. 6(d)). It is also worth noting the improvement on performance achieved when the prefetching is carried out employing the user-navigation instead of uniform probabilities. For all WOIs reported in Fig. 6, to prefetch data employing the user-navigation model achieves equal or superior performance than when prefetching employs uniform probabilities. This demonstrates the superiority of the prefetching strategy based on the user-navigation model.

The proposed scheme to prefetch data also allows that one (group of) client(s) is served with a higher quality than the others. This mechanism is commonly referred to as QoS. In this context QoS is devised so that some of the JPIP clients have a higher priority during the prefetching mode than the others. The objective is to provide better responsiveness to some premium clients of the LAN. The framework deployed by the proposed JPIP proxy server allows the introduction of QoS by multiplying the probability $P(X)$ of the potential future WOIs requested by the client with premium service by a factor selected depending on the service that the client requires. See, for example, in Fig. 6(a) the results achieved when the probabilities of client 1 are multiplied by a factor of 4. The WOIs requested by this client are, in general, already in the cache of the proxy when it requires them, so they are delivered to the client almost instantly. Evidently, this degrades the responsiveness for some of the other clients because the proxy may not prefetch data for them. QoS strategies such as these may be useful in pay-per-service scenarios or emergencies in which a rescue team is in a critical situation.

The results achieved by the proxy server using the simple prefetching (i.e., that with uniform probabilities) are not reported in the following figures to avoid cluttering them, though similar results as those reported in Fig. 6 hold.

Fig. 7 reports the results achieved when five clients browse the "Catalunya" image. We recall that -only for this imagethe first WOI transmitted by the server is situated at the top of the image. Results are reported for the proxy using the prefetching strategies based on the user-navigation model that employs probabilities $P^{\prime}(X)$ and $P^{\prime \prime}(X)$. We recall that probabilities $P^{\prime \prime}(X)$ correspond to those reported in Fig. 4(d). Results for the the client-server strategy and the conventional proxy are also reported in this figure. Again, the experimental results indicate that proxy servers that employ prefetching 


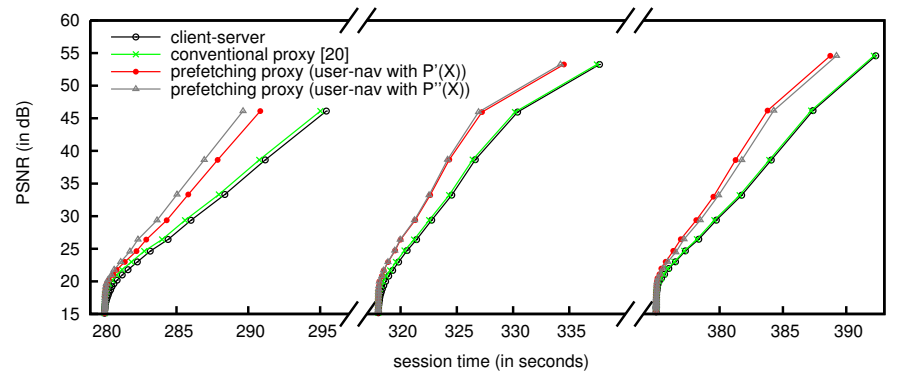

(a)

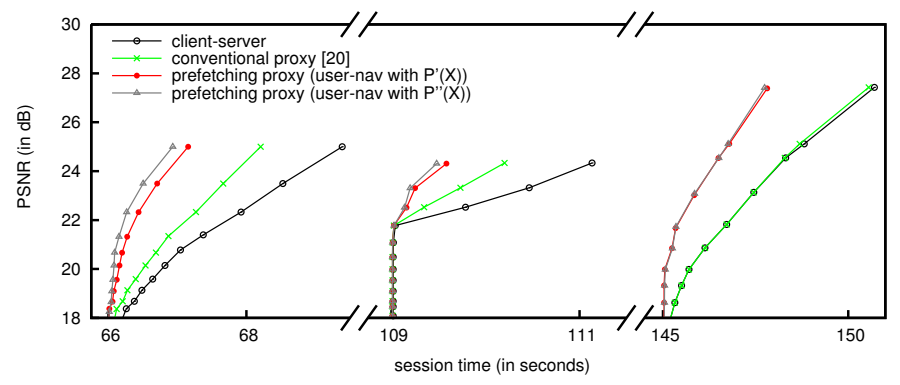

(c)

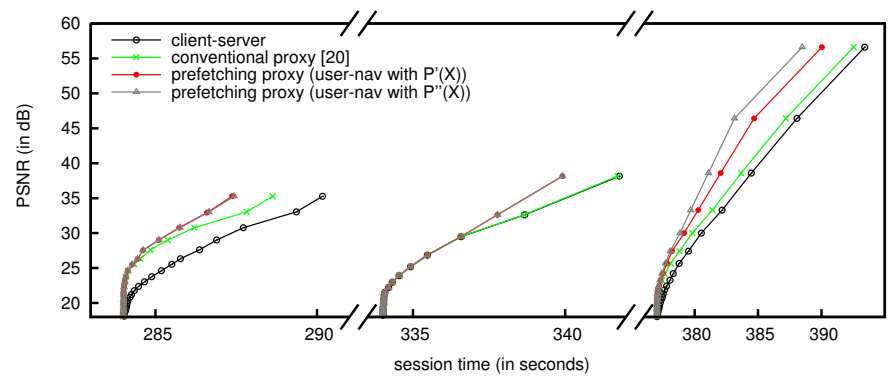

(b)

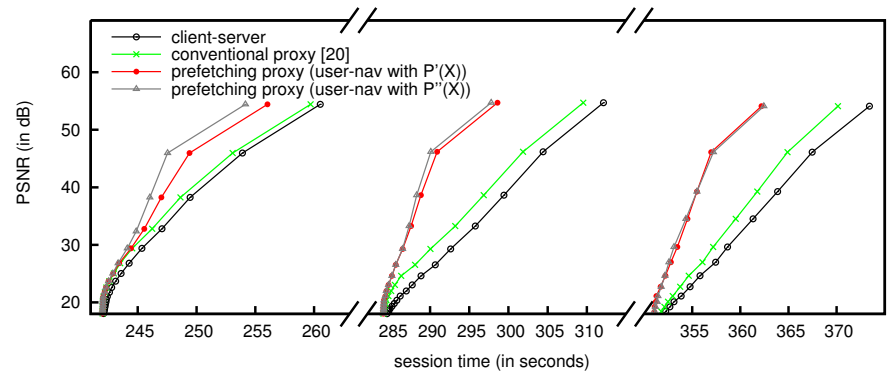

(d)

Fig. 7: Evaluation of the performance achieved by a proxy server with a prefetching strategy based on a user-navigation model extracted for the image "Catalunya" compared to three other strategies. The following WOIs are reported: (a) 8, 9, and 10 from client 1; (b) 7, 8, and 9 from client 2; (c) 2, 3, and 4 from client 4; and (d) 7, 8, and 9 from client 5.

strategies deliver WOIs to clients more rapidly than a conventional proxy server or a client-server strategy. The use of probabilities that have a major incidence on movements that go downward (i.e., $P^{\prime \prime}(X)$ ) permits the recovery of WOIs slightly faster than when probabilities of the user-navigation model are more generic (i.e., $P^{\prime}(X)$ ). For the WOIs reported in this figure, the difference between these two prefetching strategies is two seconds, at most. These results suggest that the responsiveness of clients can be enhanced by using a specific probability model for images whose first WOI transmitted is not at the center of the image, though the gains achieved when doing so are modest.

Fig. 8 reports the results achieved when four clients browse the "Port-au-Prince city" image. The prefetching strategy based on the semantic map is deployed using the regions and priorities depicted in Fig. 5. This strategy is compared to the proxy that uses prefetching based on the user-navigation model with probabilities $P^{\prime}(X)$, to a conventional proxy, and to a client-server strategy. The results achieved are similar to those reported previously. The proxy servers employing prefetching strategies achieve the best results. The prefetching strategy employing the semantic map achieves, on average, slightly better performance than that of the user-navigation model, though the differences are insignificant. For some WOIs, the use of probabilities $P^{\prime}(X)$ achieves better results than the semantic map. These results indicate that, in this context, both the user-navigation model and the semantic map are appropriate models to predict with precision the next movements of the users. To the best of our knowledge, it is not possible to combine the semantic map with the user- navigation model to create a prefetching strategy that improves these results due to the probabilities given by both strategies would interfere.

Fig. 9 reports the network statistics obtained in the experiment of Fig. 8. Only two strategies are depicted to avoid cluttering the figure, namely, the conventional proxy server, and the proxy server with prefetching based on the semantic map. The figure reports the Megabytes (MB) transmitted in the uplink and in the downlink. The uplink is the connection between the proxy and the server (i.e., $B$ in Fig. 1). It carries the requests done to the server. The proxy with prefetching transmits approximately $2 \mathrm{MB}$ in total, whereas the conventional proxy transmits $0.35 \mathrm{MB}$. These differences are caused because the proxy with prefetching requests more data when the connection is idle. Despite these differences, the amount of information transmitted by the two strategies in the uplink is very low. The downlink is the connection between the server and the proxy (i.e., $C$ in Fig. 1). It carries the codestream segments requested to server, so the amount of information transmitted in this link is much larger than that of the uplink. All the information transmitted in the downlink is kept in the cache of the proxy, so the size of the proxy cache is proportional to the data transmitted in the downlink. Again, the proxy with prefetching transmits more information than the conventional proxy because it utilizes idle times to retrieve more data. It is important to provide a large cache to the proxy to avoid removing data. In general, this is not an issue because the cache can be saved as a file in the hard drive of the proxy. The capacity of the cache in the client is not critical either because the client only receives and caches 


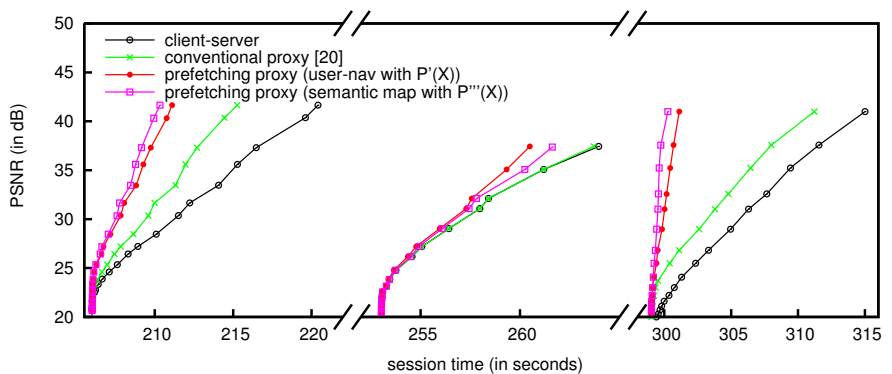

(a)

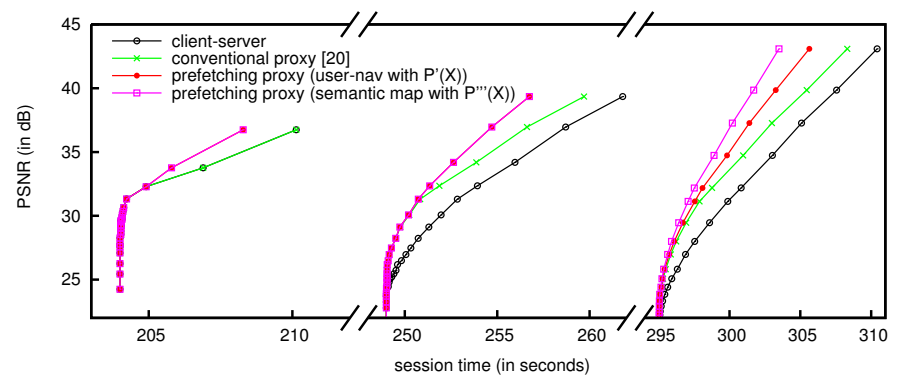

(c)

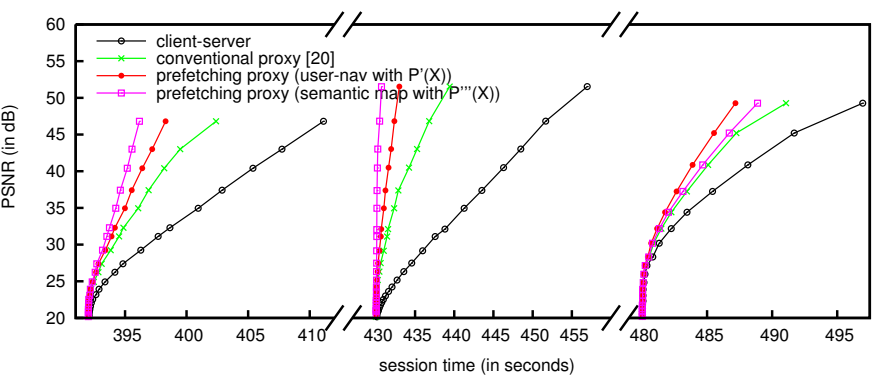

(b)

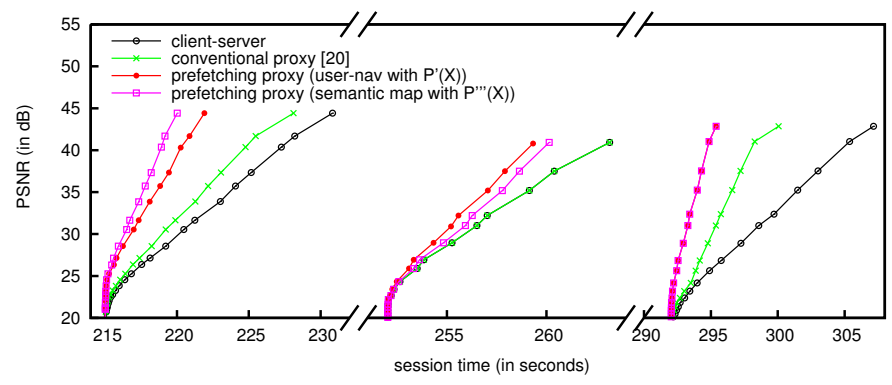

(d)

Fig. 8: Evaluation of the performance achieved by a proxy server with a prefetching strategy based on a semantic map compared to three other strategies. The image browsed is "Port-au-Prince city". The following WOIs are reported: (a) 6, 7, and 8 from client 1; (b) 10, 11, and 12 from client 2; (c) 6, 7, and 8 from client 3; and (d) 6, 7, and 8 from client 4.

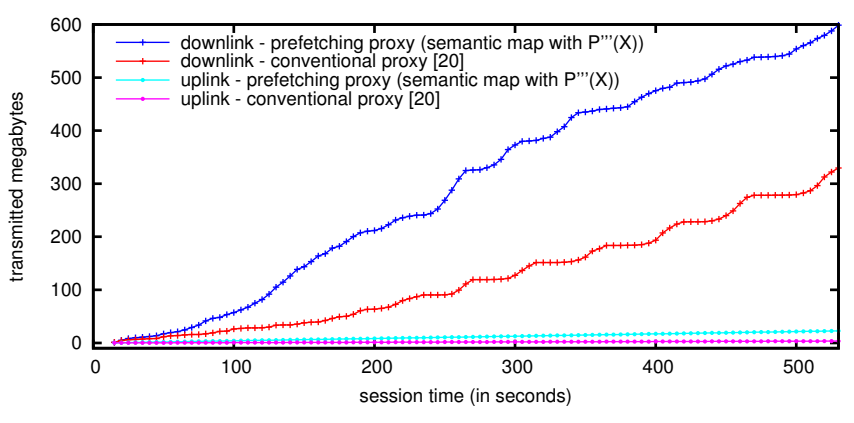

Fig. 9: Evaluation of the network statistics obtained in the same experiment as that of Fig. 8. The strategies evaluated are the conventional proxy and the proxy server with a prefetching strategy based on the semantic map.

those data corresponding to the requested WOIs. Furthermore, the cache of the client could be completely avoided by using a stateless connection between the client and the proxy.

To summarize all the results, Table I reports the average time required to transmit all layers from all WOIs requested from all clients, for the three images evaluated before. In addition to the other strategies discussed before, this table also reports the results achieved by the client-server strategy in which each client employs prefetching individually, and the strategy in which all clients prefetch individually and a conventional proxy server caches all transmitted data. On average for the three images, the response time required by the client-server strategy, the client-server strategy with prefetching at the clients, the conventional proxy, and the conventional proxy with prefetching at the clients is 11.8, 10.15, 9.31, and 7.6 seconds, respectively. The proxy server using prefetching strategies with the uniform model of probabilities achieves an average response time of 6.72 seconds, whereas the use of the user-navigation model decreases the response time to 6.19 seconds. The semantic-map based prefetching employed for the "Port-au-Prince city" image achieves virtually same results as those of the user-navigation model. These results indicate that the transmission strategies that enhance the most the responsiveness of the overall system are those that include a JPIP proxy server using prefetching strategies based on a user-navigation model or a semantic map. We remark that the average response time when using the proposed JPIP proxy server is nearly half as that needed by a client-server strategy, and approximately $1 / 3$ less than that needed by a conventional proxy. The use of prefetching at each client without any proxy achieves poor performance in these experiments, decreasing the responsiveness time of the clients in less than 1.2 seconds as compared to the average response time decrease of 5.6 seconds achieved by the proposed proxy employing the user-navigation model. The performance achieved when prefetching is carried out at each client can be improved using a conventional proxy server, though the achieved results do not outmatch those achieved when prefetching is carried out at the proxy.

\section{CONCLUSions}

This paper considers the interactive browsing of large resolution images through the JPEG2000 Interactive Protocol 
TABLE I: Evaluation of the average response time for all WOIs from all clients. Results are reported in seconds. Cells with a dash indicate that the corresponding transmission strategy does not apply on that image.

\begin{tabular}{|c|c|c|c|c|}
\hline & \multicolumn{2}{|c|}{ Port-au-Prince } & \multirow[b]{2}{*}{ Catalunya } & \multirow[b]{2}{*}{ average } \\
\hline & airport & city & & \\
\hline \multicolumn{5}{|l|}{ conventional strategies } \\
\hline client-server & 15.45 & 12.53 & 7.42 & 11.80 \\
\hline $\begin{array}{l}\text { client-server with } \\
\text { prefetching at clients }\end{array}$ & 13.88 & 10.74 & 5.82 & 10.15 \\
\hline conventional proxy [20] & 13.50 & 8.73 & 5.70 & 9.31 \\
\hline $\begin{array}{l}\text { conventional proxy with } \\
\text { prefetching at clients }\end{array}$ & 10.31 & 8.03 & 4.45 & 7.60 \\
\hline \multicolumn{5}{|l|}{ proposed strategies } \\
\hline simple [1] & 10.92 & 4.98 & 4.25 & 6.72 \\
\hline user-nav model $P^{\prime}(X)$ & 9.87 & 4.65 & 4.04 & 6.19 \\
\hline user-nav model $P^{\prime \prime}(X)$ & - & - & 3.78 & - \\
\hline semantic map $P^{\prime \prime \prime}(X)$ & - & 4.63 & - & - \\
\hline
\end{tabular}

(JPIP) when a collection of clients in a local area network (LAN) requests windows of interest (WOIs) from an image in a remote server. In this work, the capabilities of a JPIP proxy server located within the LAN are extended. Our main insight is to introduce prefetching strategies at the proxy to optimize the responsiveness of all clients simultaneously. Prefetching is carried out only during instants at which the connection between the server and the clients is idle. Key to maximize performance is to anticipate with precision the future movements of the users. Two strategies are proposed to do so. The first one is based on a user-navigation model extracted from logs collected by several image servers. The second strategy is based on the content, or the semantic, of the image. The proposed JPIP proxy server also reformulates the WOIs requested to the server so that the transmission of data between proxy and server is fully optimized. Experimental results carried out with earth observation images suggest that the proposed JPIP proxy server reduces the average response time of a conventional proxy server and a client-server strategy by $1 / 3$ and $1 / 2$, respectively. The lower response time achieved by the proposed strategy enhances the browsing experience of the end-user notably.

This work may be extended to images from other fields such as the medical or the video. Also, the models to predict future user movements might be extended to consider retrospective adaptive prefetching [31], or visual attention [32].

\section{ACKNOWLEDGMENT}

The authors thank our colleagues from CREAF for interesting discussions and suggestions, and the anonymous reviewers and associate editor for their comments and remarks.

\section{REFERENCES}

[1] J. L. Monteagudo-Pereira, F. Auli-Llinas, J. Serra-Sagrista, and J. Bartrina-Rapesta, "Smart JPIP proxy server with prefetching strategies," in Proc. IEEE Data Compression Conference, Mar. 2010, pp. $99-108$
[2] J. L. Monteagudo-Pereira, F. Auli-Llinas, J. Serra-Sagrista, A. Zabala, J. Maso, and X. Pons, "Enhanced transmission of JPEG2000 imagery through JPIP proxy and user-navigation model," in Proc. IEEE Data Compression Conference, Apr. 2012, pp. 22-31.

[3] Information technology - JPEG 2000 image coding system - Part 1: Core coding system, ISO/IEC Std. 15444-1, Dec. 2000.

[4] S. Deshpande and W. Zeng, "Scalable streaming of JPEG2000 images using hypertext transfer protocol," in Proc. ACM International Conference on Multimedia, vol. 9, Oct. 2001, pp. 372-381.

[5] D. Taubman, "Remote browsing of JPEG2000 images," in Proc. IEEE International Conference on Image Processing, Sep. 2002, pp. 229-232.

[6] J. Li and H.-H. Sun, "On interactive browsing of large images," IEEE Trans. Multimedia, vol. 5, no. 4, pp. 581-590, Dec. 2003.

[7] E. A. Politou, G. P. Pavlidis, and C. Chamzas, "JPEG2000 and dissemination of cultural heritage over the internet," IEEE Trans. Image Process., vol. 13, no. 3, pp. 293-301, Mar. 2004.

[8] R. Rosenbaum and H. Schumann, "JPEG2000-based image communication for modern browsing techniques," in Proc. SPIE International Conference on Image and Video Communications and Processing, vol. 5685, Jan. 2005, pp. 1019-1030.

[9] Information technology - JPEG 2000 image coding system Part 9: Interactivity tools, APIs and protocols, ISO/IEC Std. 15444-9, Dec. 2005.

[10] K. Krishnan, M. W. Marcellin, A. Bilgin, and M. S. Nadar, "Efficient transmission of compressed data for remote volume visualization," IEEE Trans. Med. Imag., vol. 25, no. 9, pp. 1189-1199, Sep. 2006.

[11] V. J. Tuominen and J. Isola, "Linking whole-slide microscope images with DICOM by using JPEG2000 interactive protocol," SPRINGER Journal of Digital Imaging, vol. 23, no. 4, pp. 454-462, Apr. 2010.

[12] J. Monteagudo-Pereira, J. Bartrina-Rapesta, F. Auli-Llinas, J. SerraSagrista, A. Zabala, and X. Pons, "Interactive transmission of spectrally wavelet-transformed hyperspectral images," in Proc. SPIE International Conference on Satellite Data Compression, Communication and Processing, vol. 7084, Aug. 2008, pp. 708 405,1-12.

[13] D. Muller, B. Fleck, G. Dimitoglou, B. W. Caplins, D. E. Amadigwe, J. P. G. Ortiz, B. Wamsler, A. Alexanderian, V. K. Hughitt, and J. Ireland, "JHelioviewer: Visualizing large sets of solar images using JPEG 2000," IEEE Comput. Sci. Eng., vol. 11, no. 5, pp. 38-47, Sep. 2009.

[14] F. Auli-Llinas and D. Taubman, "Optimal delivery of motion JPEG2000 over JPIP with block-wise truncation of quality layers," in Proc. IEEE International Conference on Image Processing, Oct. 2008, pp. 28562859.

[15] A. T. Naman and D. Taubman, "JPEG2000-Based scalable interactive video (JSIV)," IEEE Trans. Image Process., vol. 20, no. 5, pp. 14351449, May 2011.

[16] _ , "JPEG2000-Based scalable interactive video (JSIV) with motion compensation," IEEE Trans. Image Process., vol. 20, no. 9, pp. 26502663, Sep. 2011.

[17] D. Taubman and R. Rosenbaum, "Rate-distortion optimized interactive browsing of JPEG2000 images," in Proc. IEEE International Conference on Image Processing, vol. 3, Sep. 2003, pp. 765-768.

[18] A. Descampe, C. D. Vleeschouwer, M. Iregui, B. Macq, and F. Marques, "Prefetching and caching strategies for remote and interactive browsing of JPEG2000 images," IEEE Trans. Image Process., vol. 16, no. 5, pp. 1339-1354, May 2007.

[19] J. P. G. Ortiz, V. G. Ruiz, M. F. Lopez, and I. Garcia, "Interactive transmission of JPEG2000 images using web proxy caching," IEEE Trans. Multimedia, vol. 10, no. 4, pp. 629-636, Jun. 2008.

[20] L. Lima, D. Taubman, and R. Leonardi, "JPIP proxy server for remote browsing of JPEG2000 images," in Proc. IEEE Workshop on Multimedia Signal Processing, Oct. 2008, pp. 844-849.

[21] D. S. Taubman and M. W. Marcellin, JPEG2000 Image compression fundamentals, standards and practice. Norwell, Massachusetts 02061 USA: Kluwer Academic Publishers, 2002.

[22] F. Auli-Llinas, J. Bartrina-Rapesta, and J. Serra-Sagrista, "Enhanced JPEG2000 quality scalability through block-wise layer truncation," EURASIP Journal on Advances in Signal Processing, vol. 2010, pp. 1-11, 2010, article ID 803542.

[23] F. Auli-Llinas, A. Bilgin, and M. W. Marcellin, "FAST rate allocation through steepest descent for JPEG2000 video transmission," IEEE Trans. Image Process., vol. 20, no. 4, pp. 1166-1173, Apr. 2011.

[24] T. Richter, "Compressing JPEG 2000 JPIP cache state information," in Proc. IEEE Data Compression Conference, Apr. 2012, pp. 13-21.

[25] D. H. Lee, J. S. Kim, S. D. Kim, K. C. Kim, Y.-S. Kim, and J. Park, "Adaptation of a neighbor selection markov chain for prefetching tiled web GIS data," in Proc. International Conference on Advances in Information Systems, Oct. 2002, pp. 213-222. 
[26] S. Quinn and M. Gahegan, "A predictive model for frequently viewed tiles in a web map," Transactions in GIS, vol. 14, no. 2, pp. 193-216, Apr. 2010.

[27] D. S. Taubman, "Localized distortion estimation from already compressed JPEG2000 images," in Proc. IEEE International Conference on Image Processing, Oct. 2006, pp. 3089-3092.

[28] X. Pons. (2004) MiraMon. Geographic Information System and Remote Sensing software. Centre de Recerca Ecolgica i Aplicacions Forestals, CREAF. Bellaterra. ISBN: 84-931323-5-7. [Online]. Available: http: //opengis.uab.cat/wms/satcat/

[29] G. Camps-Valls and L. Bruzzone, "Kernel-based methods for hyperspectral image classification," vol. 43, no. 6, pp. 1351-1362, Jun. 2005.

[30] J. L. Monteagudo-Pereira. (2012) CADI. [Online]. Available: http: //www.gici.uab.cat/CADI

[31] S. Yesilmurat and V. Isler, "Retrospective adaptive prefetching for interactive web GIS applications," SPRINGER Geoinformatica, vol. 16, no. 3, pp. 435-466, Jul. 2012.

[32] Z. Li, S. Qin, and L. Itti, "Visual attention guided bit allocation in video compression," ELSEVIER Image and Vision Computing, vol. 29, pp. 114, Jan. 2011.

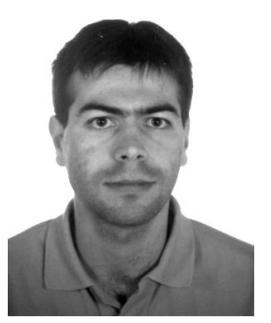

José Lino Monteagudo-Pereira recieved the B.E. and M.S. degrees in 2002 and 2004, respectively, both in Telecommunications Engineering from the Universidad de Vigo (Spain). From October 2002 to August 2004 he was with the faculty of the School of Telecommunications Engineering, Universidad de Vigo, as a researcher on image processing and multimedia. He joined the Group on Interactive Coding of Images at the Universitat Autònoma de Barcelona (Spain) in September 2004 where he is currently working towards his Ph.D. degree. Since January 2009 he has been with the Ministry of Finance and Public Administrations (Spain) as a Network and Computer Systems Administrator. His research interests lie in the areas of image compression and interactive image transmission.

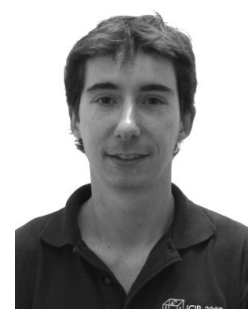

Francesc Aulí-Llinàs (S'2006-M'2008) is a Fellow of the Ramón y Cajal program funded by the Spanish Government. He is currently in the Department of Information and Communications Engineering, at the Universitat Autònoma de Barcelona (Spain). He received the B.Sc. and B.E. degrees in Computer Management Engineering and Computer Engineering in 2000 and 2002, respectively, both from the Universitat Autònoma de Barcelona (Spain), and for which he was granted with two extraordinary awards of Bachelor (awards given to the first students of the promotion). In 2004 and 2006 he respectively received the M.S. degree and the Ph.D. degree (with honors), both in Computer Science from the Universitat Autònoma de Barcelona. Since 2002 he has been consecutively awarded with doctoral and postdoctoral fellowships in competitive calls. From 2007 to 2009 he carried out two research stages of one year each with the group of David Taubman, at the University of New South Wales (Australia), and with the group of Michael Marcellin, at the University of Arizona (USA). $\mathrm{He}$ is the main developer of BOI, a JPEG2000 Part 1 implementation that was awarded with a free software mention from the Catalan Government His research interests include a wide range of image coding topics, including highly scalable image and video coding systems, rate-distortion optimization, distortion estimation, and interactive transmission, among others.

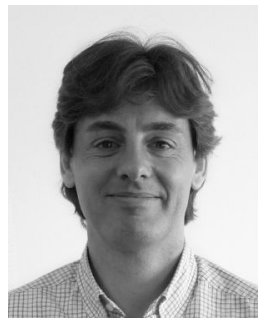

Joan Serra-Sagristà (S'97-M'05-SM'11) received the Ph.D. degree in Computer Science from Universitat Autònoma de Barcelona (UAB), Spain, in 1999. $\mathrm{He}$ is currently an Associate Professor at the Department of Information and Communications Engineering, UAB. From September 1997 to December 1998, he was at University of Bonn, Germany, funded by DAAD. His current research interests focus on data compression, with special attention to image coding for remote sensing and telemedicine applications. He serves as Associate Editor of IEEE Trans. on Image Processing and as Program Committee co-chair for IEEE Data Compression Conference. He has co-authored over one hundred publications. He was the recipient of the Spanish Intensification Young Investigator Award in 2006. 